\title{
Prozessuale Hemmnisse bei der Strafverfolgung von Stalkern
}

Matthias Krüger

\section{Einleitung}

Der Stalking-Paragraph des $\ 238$ StGB hat schon vor seiner Einführung für heftigen rechtspolitischen Wirbel gesorgt. An dieser Stelle hat die Diskussion ebenfalls getobt. Dabei kann es nicht verwundern, dass von politischer Seite „Argumente für ein strafrechtliches Stalking-Bekämpfungsgesetz “ vorgetragen wurden. ${ }^{1}$ Etwas weniger optimistisch, aber einer strafrechtlichen Lösung nicht völlig abgeneigt, ist später noch die Rede davon gewesen, dass jedenfalls die Signalwirkung des Tatbestands auf potenzielle Stalker nicht zu unterschätzen sein soll. ${ }^{2}$

Von anderer Seite wurden dagegen „Vorschläge [...] für ein sinnvolles Vorgehen gegen Stalking“ gemacht. Diskutiert wurden insofern zuvörderst Änderungen des Gewaltschutzgesetzes. ${ }^{3}$ Anderenorts versah man die „Notwendigkeit eines Stalking-Bekämpfungsgesetzes“ gleich mit einem Fragezeichen und mahnte vielmehr eine Reform des FGG-Verfahrens an. ${ }^{4}$ Sie ist inzwischen auf dem Weg. Der entsprechende Gesetzesbeschluss zur FGG-Reform in Gestalt des Regierungsentwurfs (BT-Drs. 16/6308) datiert vom 27. Juni 2008 und soll Mitte 2009 wirksam werden. Er regelt in $\$ 210$ ff. FGG n. F. unter der gesetzlichen Überschrift „Gewaltschutzsachen“ die „Verfahren nach den $\mathbb{\int} 1$ und 2 des Gewaltschutzgesetzes“. Aus Sicht der Opfer ist die Reform zu begrüßen, weil - anders als nach dem für diese Verfahren momentan noch anzuwendenden ZPO-Regeln - die Sache weitestgehend von Amts wegen zu betreiben ist (\$S $26 \mathrm{ff}$. FGG n. F. $)^{5}$, wofür freilich nach wie vor jedenfalls ein Antrag auf Erlass einer Schutzanordnung erforderlich sein wird. Von Interesse im vorliegenden Zusammenhang ist ferner noch $\$ 216 \mathrm{a}$ FG n. F. Er regelt auf Anregung des Rechtsausschusses (BT-Drs. 16/9733) - die unverzügliche „Mitteilung von Entscheidungen“ in Gewaltschutzsachen an die „zuständigen Polizeibehörden und andere öffentliche Stellen, die von der Durchführung der Anordnung betroffen sind“. Damit wird ein Vorschlag des Bundesrats aufgegriffen. ${ }^{6}$ Bislang mangelt es an einer ausreichenden Rechtsgrundlage für eine diesbezügliche Informationspflicht. Sie sei wiederum erforderlich, weil ein Informationsdefizit zwischen den Beteiligten besteht. Insbesondere die AntragstellerInnen würden davon ausgehen, dass die herbeigerufene Polizei von der Anordnung weiß. Weil die Beamten vor Ort davon aber gerade nicht Kenntnis haben müssen und vom - aus besagtem Grund diesbezüglich schweigenden - Stalking-Opfer nicht vermittelt bekommen, können sie Verstöße gegen Schutzanordnungen nicht, jedenfalls nicht wirksam der Ahndung zuführen. Durch eine regelmäßige Übermittlung der Entscheidungen seitens des Gerichts an die Polizei soll dieses Informationsgefälle beseitigt werden, damit Verstöße gegen Anordnungen nach dem Gewaltschutzgesetz zukünftig (noch) effektiver unterbunden und geahndet werden können.

Eine andere Sorge hat sich inzwischen ebenfalls auf gesetzgeberischem Wege erledigt. Die Polizei muss keine Angst mehr haben, gegen das Anwaltsmonopol zu verstoßen, wenn sie Anzeigewilligen rät, Maßnahmen nach dem Gewaltschutzgesetz zu beantragen. ${ }^{7}$ Das Rechtsdienstleistungsgesetz (RDG) vom 12. Dezember 2007 (BGBl. I S. 2840) erlaubt nunmehr in $\mathbb{8}$ Abs. 1 Nr. 2 RDG, dass Behörden im Rahmen ihres Aufgaben- und Zuständigkeitsbereichs Rechts- dienstleistungen erbringen dürfen, wozu der geschilderte Rat allemal zählen dürfte.

Ob die Summe der beiden gesetzgeberischen Aktivitäten dazu führt, „dass das Gewaltschutzgesetz in seinem Anwendungsbereich ausgeschöpft wird" 8 , bleibt aber noch abzuwarten. Selbst wenn es sich bestätigen sollte, würde sich die rechtspolitische Kritik an $\mathbb{} 238$ StGB darin nicht zwangsläufig ex post bestätigt sehen. Die Debatte hinterlässt nämlich etwas den Eindruck, dass sie sich hoffnungslos in - sich scheinbar gegenseitig ausschließende oder jedenfalls als solche dargestellte - Alternativen festgefahren zu haben scheint. Davon sollte sich man vielleicht einmal befreien und die verschiedenen Ansätze im Kampf gegen Stalker nicht als Alternativen, sondern mehr als - nebeneinander bestehende - Varianten begreifen.

\section{Erstes Zahlenmaterial zu $\ 238$ StGB}

Das Strafrecht scheint dabei aber einen eher bescheidenen Beitrag zu leisten. Jedenfalls könnten erste Zahlen diesen Schluss bei flüchtiger Betrachtung der Problematik nahe legen. Es klafft eine nicht unerhebliche Lücke zwischen der Zahl von Anzeigen einerseits und der (rechtskräftig abgeschlossenen) Strafverfahren wegen $\ 238$ StGB andererseits. Der „Weiße Ring“ etwa geht laut Spiegel-Online vom 19. Mai 2008 davon aus, dass sich im Zeitraum seit April 2007 (inzwischen mehr als) ca. 15.000-mal jemand derart belästig gefühlt hat, dass er dies bei der Polizei gemeldet hat. Allein in Berlin wurden im Jahre 2007 etwa 1000 Fälle zur Anzeige gebracht. Gegen 110 Täter sind Gerichtsverfahren anhängig.

Inzwischen liegen daneben noch erste amtliche Zahlen vor. Die Polizeiliche Kriminalstatistik (PKS) von 2007 weist bei den Straftaten gegen die persönliche Freiheit gegenüber 2006 eine Zunahme um 9,6\% auf 182.219 Fälle aus. Für den Anstieg dürfte insbesondere die Einführung des Straftatbestands der Nachstellung (Stalking) gemäß \238 StGB verantwortlich sein. 2007 konnten 11.401 Fälle dieser Art verzeichnet werden. Zu berücksichtigen ist aber, dass die Vorschrift nicht bloß erst im Laufe des Jahres 2007 in Kraft trat, sondern überdies die Erfassung in einem eigenständigen Straftatenschlüssel noch nicht in allen Bundesländern realisiert werden konnte. ${ }^{9}$ In Sachsen jedenfalls belief sich die Zahl der Opfer in diesem Zusammenhang auf 777 Personen. ${ }^{10}$ Die Zahlen des „Weißen Rings“ sehen sich vor diesem Hintergrund durchaus bestätigt.

Die Verurteiltenstatistik für 2007 wird dagegen, ohne prophetisch veranlagt zu sein, voraussichtlich eine andere Sprache sprechen. Die Verurteilungen nach $\$ 238 \mathrm{StGB}$, wenn es sie überhaupt gibt, dürften im Promille-Bereich liegen. Damit könnten Pessimisten, wie sie bei Spiegel-Online zu Wort gekommen sind, Recht behalten. Anwälte und Beratungsstellen warnen danach die Betroffenen schon lange davor, nicht allzu große Hoffnungen in den neuen Straftatbestand zu setzen. Der Anti-Stalking-Paragraph gehört für sie zu den „Halbstarken unter den Rechtsinstrumenten“: große Worte aber nichts dahinter. In Berlin hat die Polizei auf diese „Unzulänglichkeiten des Strafrechts“ reagiert und eine erste Beratungsstelle in Deutschland eröffnet, die sich an die Täter wendet und um diese kümmert. Im Übrigen aber sende die Justiz ein „fatales Signal“ aus, wenn Stalker 
nicht verurteilt würden. Schließlich seien Urteile, die abschrecken könnten, bislang eher selten. Bezeichnenderweise ist in dem berichteten Fall wohl nicht aus $\$ 238 \mathrm{StGB}$, sondern vielmehr wegen Verstoßes gegen das Gewaltschutzgesetz zu einer Bewährungsstrafe verurteilt wurden, die später widerrufen worden ist, weil der Verurteilte offenbar entweder gegen Bewährungsauflagen oder erneut gegen das Gewaltschutzgesetz verstoßen hat.

\section{Rechtspolitische Kritik an $\mathbb{} 238$ StGB}

In diesen geringschätzigen Werturteilen und der - bislang jedenfalls - geringen Zahl von Verurteilungen scheinen sich kritische Stimmen aus der Strafrechtswissenschaft bestätigt zu sehen. Sie machen in \238 StGB ein „weiteres Exempel für strafrechtlichen Aktionismus“ aus ${ }^{11}$, worin sich zugleich ein unverkennbarer „Hang zu einem perfektionistischen Strafrecht“ zeigen soll ${ }^{12}$. Seine Existenz verdanke er einzig und allein einem bloßen „Akt symbolischer Gesetzgebung “13, der sich keinesfalls in der materiell-rechtlichen Regelung des $\mathbb{2} 238$ StGB erschöpfen soll, sondern die Einführung der sog. Deeskalationshaft in $\$ 112$ a Abs. 1 Nr. 1 StPO betreffe, die „durchaus in die Nähe symbolischer Gesetzgebung gestellt werden“ könne. ${ }^{14}$ Ein neues strafbewehrtes Verbot müsse aber mehr erbringen, „als das (trügerische) Gefühl, sinnvoll tätig geworden zu sein“, monieren etwa noch Kinzig/Zander, die zugleich „Zweifel hinsichtlich der kriminalpolitischen Notwendigkeit einer neuen Vorschrift $\ 238$ StGB im Kernstrafrecht" äußern. ${ }^{15}$ Sehr pointiert hat Eiden diese mehr rechts- und kriminalpolitische Kritik wie folgt zusammengefasst: Der Gesetzgeber habe „wieder einmal seiner Straflust gefrönt und einen neuen Tatbestand in das Kernstrafrecht hineingeflochten. Der Keil, den er zugleich mit ungeschliffener Axt aus dem noch weiß gebliebenen Bereich menschlichen Handelns herausgetrennt hat, trägt den harmlosen Namen Nachstellung. " 16 Angesichts dieser düsteren Prognosen und (Vor-)Urteile sowie der - diese womöglich bestätigenden - bislang geringen Zahl von Verurteilungen nach $\$ 238$ StGB stellt sich die Frage, ob man das strafrechtliche Schwert im Kampf gegen Stalker strecken sollte.

Wenngleich man sicher nicht ausschließlich auf das strafrechtliche Pferd setzen sollte, um Stalkern Herr zu werden, ist es für einen Abgesang an die neue Strafvorschrift viel zu früh. Bisherige Statistiken sind schon deshalb kaum aussagekräftig, weil wegen „beharrlich“ schon materiell-rechtlich etwas Zeit vergehen muss, bevor wegen \238 StGB ermittelt werden kann. Weil diese Ermittlungen wiederum, selbst wenn man sie beharrlich betreibt, ebenfalls (geraume) Zeit in Anspruch nehmen, ist es nicht verwunderlich, dass Verurteilungen aus $\$ 238$ StGB bislang selten sind. Es ist nicht ausgeschlossen, dass es schon in einem Jahr anders aussieht. $\$ 303$ Abs. 2 StGB jedenfalls hat - im Herbst 2005 eingeführt - im Kampf gegen Graffiti im Jahre 2006 eher noch ein Schattendasein mit bloß 217 Verurteilungen bei 352 Gerichtsverfahren geführt. ${ }^{17}$ Ohne Kaffeesatzleserei betreiben zu wollen, dürften die Zahlen im Jahre 2007 höher ausfallen. Zumindest dieselbe Zeit sollte $\$ 238$ StGB gegeben werden, bevor man ein erstes (Zwischen-)Urteil ob seiner (Un-)Wirksamkeit im Kampf gegen Stalker fällt.

Im Übrigen ist auf ein Urteil des LG Kiel aufmerksam zu machen. ${ }^{18}$ Im Rahmen eines Nachbarschaftsstreits hat der - wegen Körperverletzung und Betrugs ${ }^{19}$ - angeklagte und später verurteilte Täter in zahlreichen Fällen bei Unternehmen angerufen und diese mit der Lieferung von Waren bzw. Erbringung von Dienstleistungen beauftragt, „wobei er (der Angekl.) die Absicht hatte, den Nebenkläger [seinen Nachbarn] dadurch in dauerhafte Aufregung und Unruhe zu versetzen. Um dies zu erreichen, kam es dem Angekl. darauf an, dass die beauftragten Unternehmen die Wohnanschrift des Nebenkl. auch aufsuchten, um ihre vertraglichen Leistungen zu erbringen bzw. anzubieten. Wie für den Angekl. vorhersehbar, wurde der Nebenkl. durch die [...] Tätigkeiten der Unternehmen tatsächlich massiv in Angst und Schrecken versetzt, so dass sich bei ihm Unruhezustände, Nervosität und Schlafstörungen einstellten; letztere äußerten sich insbesondere durch massive Einschlafstörungen des Nebenkl. Insgesamt verschlechterte sich der Gesundheitszustand des Nebenkl. so, dass dieser sich [...] in ärztliche Behandlung bei seinem Hausarzt begab und mit einem Psychopharmakon behandelt wurde. "Ob es sich in soziologischer Hinsicht bei einem solchen Nachbarschaftszwist (noch) um einen Fall von Stalking handelt, sollen besser andere, hierzu berufenere Professionen beurteilen. Aus juristischer Perspektive hätte $\$ 238 \mathrm{StGB}$ - de lege lata ${ }^{20}$ - jedenfalls nicht völlig fern gelegen, wenn das Urteil nicht bereits am 3. 3. 2006 gefällt worden wäre, sondern sich der Fall erst im Jahre 2007 zugetragen hätte. $\mathrm{Ob}$ in der Einnahme des Psychopharmakons nicht bloß eine - in mittelbarer Täterschaft kraft des Opfers als Werkzeug gegen sich selbst begangene $^{21}$ - Körperverletzung, sondern zugleich eine „schwerwiegende Beeinträchtigung der Lebensgestaltung“ erblickt werden könnte, insbesondere wenn sie über einen längeren Zeitraum erfolgt wäre $^{22}$, hätte jedenfalls einer näheren Prüfung bedurft.

\section{Inhaltliche Kritik an $\ 238 \mathrm{StGB}$}

Damit ist man bei einem wunden Punkt des $\$ 238$ StGB angelangt. Es werden materiell-rechtliche Hemmnisse in der Anwendung der Vorschrift darin gesehen, dass sie mit „vagen Formulierungen“ gespickt sei, wie es bei Spiegel-Online heißt. Das Schrifttum teilt diese Vorbehalte. Kinzig/Zander etwa machen eine „Fülle von unbestimmten Rechtsbegriffen“ aus und führen dafür „beharrlich “, „räumliche Nähe“, „sonstige Mittel“, „andere vergleichbare Handlung“ und „schwerwiegende Beeinträchtigung “ an, die teilweise sogar miteinander kombiniert werden würden. ${ }^{23}$ Diese Kritik hat Freudenberg bereits während des Gesetzgebungsverfahrens zu $\$ 238$ StGB artikuliert. Danach enthält die Vorschrift „unbestimmte Rechtsbegriffe in wechselseitiger Abhängigkeit mit unklarer Rangbestimmung, auf die schließlich eine generalklauselartige Alternative Bezug nimmt“". ${ }^{24}$ Dass es sich bei manchen Formulierungen des $\$ 238$ StGB um ausfüllungsbedürftige (Rechts-)Begriffe hält, lässt sich in der Tat schwerlich leugnen.

Die Frage ist freilich, ob es sich insofern um unüberwindbare Hürden bei der Anwendung des $\mathbb{} 238$ StGB handelt. Dabei sollen an dieser Stelle nicht eigene, frühere Bemühungen um die Auslegung der genannten „vagen Formulierungen“ erneut unternommen - oder gegen zwischenzeitlich erfolgte Kritik verteidigt - werden. ${ }^{25}$ Vielmehr soll bloß auf $\$ 303$ Abs. 2 StGB aufmerksam gemacht werden. Er steht ebenfalls unter dem Verdacht, sehr vage formuliert zu sein. ${ }^{26}$ Wenn man die Probleme insofern in den Griff zu bekommen scheint, sollte man bei $\$ 238$ StGB ebenfalls nicht vorschnell den Kopf in den Sand stecken.

\section{Ausgestaltung von $\mathbb{2 3 8}$ Abs. 1 StGB als (relatives) Antrags- und Privatklagedelikt}

Damit stellt sich die vordringliche Frage, ob es prozessuale Hemmnisse bei der Strafverfolgung von Stalkern gibt und wie diese gegebe- 
nenfalls aus dem Weg zu räumen sind, sei es durch gesetzgeberische Aktivitäten, sei es durch Änderung der Praxis der Strafverfolgungsbehörden. Dabei erweist sich ausnahmsweise einmal nicht, obwohl es verschiedentlich angenommen wird ${ }^{27}$, das Opportunitätsprinzip, wie es de lege lata insbesondere in $\$ \mathbb{S} 153,153$ a StPO niedergelegt ist, als diesbezüglich entscheidendes Hemmnis. Bei Privatklagedelikten, wozu das sog. milde Stalking zählt, kann nämlich nach $\ 153 \mathrm{a}$ StPO, nicht aber nach $\$ 153$ StPO verfahren werden ${ }^{28}$. Damit gerät zwangsläufig $\ 238$ Abs. 4 StGB in den Blick, wonach der StalkingGrundtatbestand bloß auf Antrag des Verletzten verfolgt wird, es sei denn, dass ein besonderes öffentliches Interesse die Strafverfolgung von Amts wegen gebietet. Dies korrespondiert mit $\$ 374$ Abs. 1 Nr. 5 StPO, der $\$ 238$ Abs. 1 StGB unter die Privatklagedelikte einordnet, bei denen die öffentliche Anklage bekanntlich bloß im öffentlichen Interesse erfolgt ( $\$ 376$ StPO). Diese Kombination könnte sich als ein gravierendes prozessuales Hemmnis im strafrechtlichen Kampf gegen Stalking erweisen. Entsprechende Vorbehalte wurden bereits vor Inkrafttreten des $\mathbb{2} 238$ StGB geäußert. Es sei angesichts dieser Ausgestaltung „gut möglich, dass die Gesetzesänderung nicht zu einer erhöhten Zahl an Verurteilungen führen wird“. 29

\section{Allgemeines zum (besonderen) öffentlichen Interesse}

Damit läuft es auf die Frage hinaus, wie das (besondere) öffentliche Interesse im Allgemeinen und bei Stalking im Speziellen zu verstehen ist. Dabei gilt es, zunächst das Verhältnis zwischen dem „,besonderen öffentlichen Interesse“ einerseits und dem (einfachen) „öffentlichen Interesse“ andererseits zu beleuchten. Wenngleich es noch nicht endgültig geklärt ist ${ }^{30}$, lässt sich Folgendes wohl kaum wegdiskutieren: Wenn das „besondere öffentliche Interesse“ im Sinne von $\mathbb{} 238$ Abs. 4 StGB zu bejahen ist, lässt sich das (einfache) „öffentliche Interesse" bei der Entscheidung gemäß \376 StPO wohl schwerlich in Abrede stellen. Damit wird die Vorschrift nicht etwa in der Weise überflüssig, als dass das öffentliche Interesse durchweg anzunehmen ist. Dies würde schon seinem gesetzlichen Ausnahmecharakter zuwiderlaufen („nur“). Im Übrigen darf Folgendes nicht übersehen werden: Wenn das Ermittlungsverfahren bei einem Privatklagedelikt durch Strafantrag des Verletzten in Gang gesetzt worden ist, bleibt es bei der Filterfunktion des $\$ 376$ StPO. Wenn aber schon das „besondere öffentliche Interesse“ von den Strafverfolgungsbehörden im Rahmen des Vorverfahrens angenommen worden ist, dürfte es am (einfachen) öffentlichen Interesse für ein amtswegiges Einleiten des Hauptverfahrens regelmäßig nicht fehlen.

Im Übrigen schweigt sich das Strafgesetz selbst jedoch dazu aus, wie die Begriffe zu verstehen sind. In den Richtlinien zum Straf- und Bußgeldverfahren (RiStBV) sind sie dagegen näher umschrieben. Als Verwaltungsvorschriften sind diese für Staatsanwaltschaften bindend. Dies gilt hingegen nicht für Gerichte, allerdings bloß unmittelbar. In gewisser Weise sind sie dagegen sehr wohl, jedenfalls im vorliegenden Zusammenhang konstitutiv für Strafgerichte. Das „,besondere öffentliche Interesse " unterliegt zwar nach einer im Vordringen begriffenen Auffassung der (straf-)gerichtlichen Überprüfung ${ }^{31}$, nicht aber nach der - verfassungsgerichtlich abgesegneten - h. M. in Rechtsprechung und Schrifttum ${ }^{32}$. In dieser Hinsicht schlagen die Richtlinien gewissermaßen auf das gerichtliche Verfahren durch.

\section{Bestrebungen zur Reform der Richtlinien zum Straf- und Bußgeldverfahren aus Anlass der Einführung von $\int 238$ StGB}

Damit erhebt sich die Frage, ob angesichts dieser Richtlinien auf einen Strafantrag des Opfers verzichtet und stattdessen das Verfahren von Amts wegen betrieben werden kann - und sollte. Von der bündnisgrünen Bundestagsfraktion ist dies auf Grundlage der geltenden Richtlinien zum Straf- und Bußgeldverfahren in Zweifel gezogen worden. In einem Entschließungsantrag im Rahmen des Gesetzgebungsverfahrens zu $\$ 238$ StGB hat sie die Bundesregierung vielmehr aufgefordert, sich für eine sachgerechte bundeseinheitliche Behandlung von Strafverfahren wegen Stalkings einzusetzen, „indem sie gegenüber den JustizministerInnen und -senatorInnen darauf hinwirkt, dass die Richtlinien für das Straf- und Bußgeldverfahren (RiStBV) dahingehend ergänzt werden, dass das besondere öffentliche Interesse an der Strafverfolgung insbesondere bei engen persönlichen Beziehungen oder bei einer sonstigen Unzumutbarkeit der Strafantragstellung durch das Opfer anzunehmen ist und diese Richtlinien in der Praxis konsequent umgesetzt werden ". ${ }^{33}$ In der Begründung zum Antrag heißt es noch wie folgt ${ }^{34}$ :

„Sinn und Zweck eines Straftatbestandes gegen Stalking ist ein besserer Rechtsgüterschutz für die Opfer. Dies kann aber nur bei konsequenter Strafverfolgung gelingen. Die Koalition hat sich dafür entschieden, Stalking im Grundtatbestand nicht als Offizialdelikt auszugestalten, bei dem die Staatsanwaltschaft in jedem Fall von Amts wegen ermitteln müsste. Die Tat soll vielmehr nur auf Antrag verfolgt werden, es sei denn, dass die Strafverfolgungsbehörde ein besonderes öffentliches Interesse an der Strafverfolgung annimmt. Wird die Tat damit [...] als so genanntes relatives Antragsdelikt ausgestaltet, muss jedoch dafür gesorgt werden, dass die besonderen Umstände berücksichtigt werden, die dazu führen, dass es für das Opfer - etwa aufgrund der engen persönlichen Beziehung zwischen den Beteiligten oder weil der Täter Druck ausübt - unzumutbar sein kann, Strafantrag zu stellen. Dieses Phänomen ist insbesondere aus dem Bereich der häuslichen Gewalt bekannt. Damit Stalking-Opfer nicht von der Staatsanwaltschaft allein gelassen werden, indem das besondere öffentliche Interesse verneint wird, muss dem mit Hilfe der Richtlinien für das Straf- und Bußgeldverfahren entgegengewirkt werden."

Mit dieser Begründung hat sich die bündnisgrüne Fraktion im Bundestag für eine Änderung der Richtlinien zum Straf- und Bußgeldverfahren stark gemacht. In der abschließenden Plenardebatte hat es deren Rednerin noch einmal als „besonders wichtig“ bekräftigt. ${ }^{35}$ Aber schon die linke Opposition hat darauf entgegnet, dass diese beantragte Änderung nicht hilft, „denn das meiste steht bereits in den Richtlinien, gerade hinsichtlich des öffentlichen Interesses“. ${ }^{36}$ Erst Recht verwundert es nicht, dass eine Rednerin der Koalitionsfraktionen den Entschließungsantrag für überflüssig gehalten hat. ${ }^{37} \mathrm{Da}$ mit wird implizit angenommen, dass eine Änderung der Richtlinien für das Straf- und Bußgeldverfahren nicht erforderlich ist, weil sie den von den Bündnisgrünen vorgetragenen Aspekten ohnehin schon hinreichend Rechnung tragen (würden). Wenngleich man darin wohl (noch) keinen verbindlichen Willen des Gesetzgebers erblicken kann, weil es sich bloß um die (Einzel-)Meinung von Parlamentariern handelt, darf dieser Umstand bei der Frage nicht außer Acht gelassen werden, ob bereits die geltenden Richtlinien zum Straf- und Bußgeldverfahren in Fällen von Stalking regelmäßig dazu führen, dass das (besondere) öffentliche Interesse anzunehmen ist. 


\section{Kritik an der Ausgestaltung des $\mathbb{2} 238$ Abs. 1 StGB als (relatives) Antrags- und Privatklagedelikt}

Wenn es sich in diesem Sinne verhalten würde, hätte sich zugleich die Kritik erledigt, die während des Gesetzgebungsverfahrens an \238 Abs. 4 StGB und der Ergänzung des \374 Abs. 1 Nr. 5 StPO um $\mathbb{} 238$ Abs. 1 StGB laut geworden ist. Die „Ausgestaltung des Straftatbestands als Antragsdelikt und Privatklagedelikt erscheint inkonsequent und konterkariert den im Zusammenhang mit der Verfolgung von häuslicher Gewalt mühsam errungenen Paradigmenwechsel, wonach die Verfolgung von Straftaten im privaten Bereich nicht Privatsache, sondern ein Anliegen der Gesellschaft ist. Dieser Paradigmenwechsel war erforderlich geworden, weil bis dahin die Taten im privaten Bereich durch die Staatsanwaltschaft nicht verfolgt, sondern die verletzten Personen auf den Privatklageweg verwiesen wurden. Dies ist weder den Opfern von häuslicher Gewalt noch den Opfern von Stalking zuzumuten. Zugleich wird der in Zusammenhang mit der Verfolgung häuslicher Gewalt durch die Staatsanwaltschaft erreichte Fortschritt durch eine solche Regelung massiv gefährdet. “38 Anderenorts teilt man diese Vorbehalte nicht und hält - wie schon der Gesetzgeber ${ }^{39}$ - die mit der Einstufung als Antrags- und Privatklagedelikt verbundene Teilprivatisierung aus Opferschutzgründen für sinnvoll. ${ }^{40}$ Sie wird dadurch ausgeglichen, dass eine Strafverfolgung von Amts wegen wiederum zu erfolgen hat, wenn sich das Opfer durch eine strukturelle persönliche Abhängigkeit und Unterlegenheit im Verhältnis zum Täter an der Strafantragstellung gehindert sieht bzw. aus Angst oder Furcht vor dem Täter davon absieht. ${ }^{41}$ Dies mag in der Sache durchaus zutreffend sein. Eine Subsumtion unter den Begriff des „besonderen öffentlichen Interesses“, wie er in den Richtlinien zum Straf- und Bußgeldverfahren niedergelegt ist, ist damit aber noch nicht verbunden.

Damit hat sich Buettner näher befasst. ${ }^{42}$ Sein Resümee ist für Stalking-Opfer niederschmetternd. Mit dem Antragserfordernis in $\ 238$ Abs. 4 StGB habe der „Gesetzgeber die Chance vertan, den Opfern von Nachstellungen effektiveren Schutz zu gewährleisten“. Von daher sei es „zu wünschen, dass der Gesetzgeber diese Beschränkung der Strafverfolgung aufhebt“. „Der durchaus taugliche Ansatz, den Schutz der Opfer durch die Strafverfolgungsbehörden zu verbessern, sieht sich durch die Ausgestaltung des $\$ 238$ I StGB als Privatklagedelikt seiner Wirkung beraubt, da die Verfolgung durch die Staatsanwaltschaft wegen $\mathbb{} 376$ StPO nur dann geschieht, wenn hieran auch ein öffentliches Interesse besteht. “ Dieser Widerspruch sei bloß durch „die Einordnung des $\$ 238$ I StGB in die Reihe der Offizialdelikte durch die Rücknahme der Änderungen des $\$ 374$ I Nr. 5 StPO zu beheben“. In dieselbe Richtung ging ein Änderungsantrag der bündnisgrünen Bundestagsfraktion während des Gesetzgebungsverfahrens zu $\$ 238$ StGB. Danach sollte der Stalking-Grundtatbestand nicht in den Katalog des $\$ 374$ StPO aufgenommen werden. Zur Begründung heißt es, dass es nicht wünschenswert ist, „dass Opfer von Nachstellungen auf den Privatklageweg verwiesen werden und damit ihren strafrechtlichen Schutz selbst erkämpfen müssen. Vielmehr begründet die besondere Schutzbedürftigkeit der betroffenen Opfergruppen gerade regelmäßig ein öffentliches Interesse an der Strafverfolgung " 43 , ohne dass freilich dargetan wird, ob diesem Umstand nicht bereits die geltenden Richtlinien zum Straf- und Bußgeldverfahren hinreichend Rechnung tragen. Das Verdikt der ungenutzten Chance wie der Ruf nach (gesetzlichen) Änderungen setzen aber voraus, dass eine Strafverfolgung von Amts wegen bei Fehlen und Ausbleiben eines Strafantrags tatsächlich in der Regel nicht möglich sein soll.

\section{Auslegung des „öffentlichen Interesses“ in Stalking- Fällen}

Dies versucht Buettner damit zu begründen, dass der Begriff des öffentlichen Interesses „die Verletzung des Rechtsfriedens jenseits des Lebenskreises des Verletzten durch die Tat verlangt “44 - und dies beim Stalking-Grundtatbestand regelmäßig nicht der Fall sein soll. Daran ist richtig, dass Nr. 86 Abs. 2 RiStBV in seinem Satz 1 für ein öffentliches Interesse voraussetzt, dass „der Rechtsfrieden über den Lebenskreis des Verletzten hinaus gestört und die Strafverfolgung ein gegenwärtiges Anliegen der Allgemeinheit ist, z. B wegen des Ausmaßes der Rechtsverletzung, wegen der Rohheit oder Gefährlichkeit der Tat, der niedrigen Beweggründe des Täters oder der Stellung des Verletzten im öffentlichen Leben“. Wenn es damit sein Bewenden hätte, wäre ein Strafantrag insbesondere entbehrlich, wenn Prominente Opfer von Stalking werden. Wenngleich diese Fälle in der medialen Gesellschaft besondere Aufmerksamkeit erregen, handelt es sich dennoch nicht um die überwiegend vorkommenden Fälle von Stalking. Etwa die Hälfte der Täter wird vielmehr als sog. rejected bzw. zurückgewiesener Stalker tätig ${ }^{45}$, der eine Trennung nicht verwindet und den Ex-Partner tyrannisiert ${ }^{46}$. Diese Opfer sollen nach Buettner allein gelassen werden.

Mitnichten ist es aber der Fall. Nr. 86 Abs. 2 RiStBV verzichtet in seinem Satz 2 nämlich darauf, dass das öffentliche Interesse zwangsläufig „die Verletzung des Rechtsfriedens jenseits des Lebenskreises des Opfers“ verlangt ${ }^{47}$, worauf Buettner $\$ 376 \mathrm{StPO}$ zu reduzieren scheint. Selbst wenn „der Rechtsfrieden über den Lebenskreis des Verletzten hinaus nicht gestört worden [ist], so kann ein öffentliches Interesse auch dann vorliegen, wenn dem Verletzten wegen seiner persönlichen Beziehung zum Täter nicht zugemutet werden kann, die Privatklage zu erheben, und die Strafverfolgung ein gegenwärtiges Anliegen der Allgemeinheit ist". Damit haben im Ergebnis jene Stimmen Recht, die ein - besonderes ${ }^{48}$ - öffentliches Interesse annehmen wollen, wenn sich das Opfer durch eine strukturelle persönliche Abhängigkeit und Unterlegenheit im Verhältnis zum Täter an der Strafantragstellung gehindert sieht bzw. aus Angst oder Furcht vor dem Täter davon absieht. ${ }^{49}$ Ein anderes Ergebnis - im Sinne von Buettner - hätte überdies die missliche Konsequenz, dass die Stellung des Strafantrags vom (rejected) Stalker nachgerade als Einladung zu weiteren Stalking-Aktivitäten mit dem Ziel (miss-)verstanden werden kann, den Strafantrag wieder zurückzunehmen. ${ }^{50}$ Dies sollte ebenfalls nicht unbedacht bleiben und das (besondere) öffentliche Interesse in solchen Fällen von daher regelmäßig angenommen werden.

\section{Schutz von Stalking-Opfern im Strafprozess}

Eine Beeinflussung bzw. Einflussnahme des Täters auf den Gestalkten kann aber noch unmittelbar im Strafprozess selbst erfolgen, indem der Angeklagte dem Opfer „nahe legt“, auf eine belastende Aussage zu verzichten. Wenn es diesem Druck nachgibt, könnte darin ein gravierendes Hemmnis bei der strafrechtlichen Verfolgung von Stalkern erblickt werden.

\section{Verlesen eines sog. Stalking-Tagbuchs}

Eine solche Drohung würde allerdings ins Leere gehen, wenn auf die Aussage des Opfers verzichtet und der Angeklagte auf andere Weise des Stalkings überführt werden könnte. Geradezu prädestiniert dafür wäre die Verlesung von Aufzeichnungen, in welchen das Opfer die Aktivitäten des Stalkers auflistet. Eine entsprechende 
Empfehlung, nämlich ein sog. Mobbing-Tagebuch zu führen, wird Opfern von Mobbing gegeben. ${ }^{51}$ Ohne an dieser Stelle hinterfragen zu wollen, worin die gemeinsame Schnittmenge und worin die Unterschiede zwischen beiden Phänomenen bestehen ${ }^{52}$, ist ein solcher Tipp für Stalking-Opfer ebenfalls nahezu unentbehrlich. Die einschlägige nichtjuristische Fachliteratur zu Stalking rät jedenfalls dazu. Im Stalkingtagebuch sollte jeder Vorfall, jedes Auflauern und Verfolgen, jede Kommunikation genau mit Datum, Uhrzeit, Ort und etwaigen Zeugen aufgeschrieben werden. ${ }^{53}$ Dies ist „oft unabdingbare Voraussetzung für die Einleitung von polizeilichen oder gerichtlichen Maßnahmen“. ${ }^{54}$ Aus materiell-strafrechtlichen Überlegungen erscheint es durchaus angebracht. Wenn man für „beharrlich“ eine Relation zwischen der Häufigkeit und Intensität der einzelnen Verhaltensweisen einerseits und deren Gesamtdauer andererseits verlangt ${ }^{55}$, lässt sich dies eher durch Aufzeichnungen als durch das bloße Erinnerungsvermögen des Opfers rekonstruieren. Wenn ein Stalking-Tagebuch in dieser Hinsicht materiell-rechtlichen Zwecken dienen kann, kommt nahezu zwangsläufig die Frage auf, ob es im Prozess ebenfalls dadurch dienstbar gemacht werden kann, dass man es im Rahmen der Beweisaufnahme verliest.

\section{a) Vorbehalte aus dem Verfassungsrecht}

Verfassungsrechtliche Einwände gegen eine solche Vorgehensweise bestehen nicht. Es dürfen Tagebücher zwar bloß bei der Verfolgung und Aburteilung von schweren Straftaten verlesen werden ${ }^{56}$, wozu $\$ 238$ Abs. 1 StGB sicher nicht zählt. Unter Tagebüchern in diesem Sinne versteht man aber Aufzeichnungen von „ausschließlich höchstpersönlichen Charakter“, die „eine offene, von keiner Rücksichtnahme sich selbst gegenüber beeinflusste Wiedergabe bestimmter Gemütszustände sowie Reflexionen über die eigene Persönlichkeitsstruktur“ des Verfassers und damit seine „Auseinandersetzung mit dem eigenen Ich “ betreffen. ${ }^{57}$ Darunter fallen Mobbing- oder Stalking-Tagebücher aber gerade nicht, weil sie ausschließlich - zumal von Dritten geschaffene - Fakten zum Inhalt haben.

\section{b) Vorbehalte wegen des strafprozessualen Unmittelbar- keitsgrundsatzes aus $\mathbb{S} 250$ StPO}

Der strafprozessuale Unmittelbarkeitsgrundsatz könnte aber einer Verlesung solcher Stalking-Tagebücher entgegenstehen. Er gebietet - de lege lata ${ }^{58}$ - gemäß $\$ 250$ StPO, dass Zeugen über deren Wahrnehmungen grundsätzlich in der Hauptverhandlung selbst zu vernehmen sind und dies „,nicht durch Verlesung des über eine frühere Vernehmung aufgenommenen Protokolls oder einer schriftlichen Erklärung ersetzt werden “ darf. Damit stellt sich die Frage, ob das Stalking-Tagebuch als „schriftliche Erklärung“ im Sinne der Vorschrift anzusehen ist und angesichts dessen bloß unter den Voraussetzungen des $\$ 251$ StPO verlesen werden darf. Nach der überwiegenden, insbesondere von den Strafgerichten vertretenen Auffassung sind „schriftliche Erklärungen“ im Sinne von $\$ 250$ Satz 2 StPO lediglich diejenigen Schriftstücke, die von vornherein zu Beweiszwecken verfasst worden sind. ${ }^{59}$ Dazu sollen Tagebücher zwar nicht zählen. ${ }^{60}$ Damit gemeint ist aber das Tagebuch im klassischen Sinne der Tagebuch-Entscheidungen von BGH und BVerfG, nicht hingegen das Stalking-Tagebuch. Es wird gerade zu dem Zweck geführt, den Täter zu überführen, und damit zu Beweiszwecken. Von daher fällt es unter das Verlesungsverbot des $₫ 250$ Satz 2 StPO.

Insofern bedürfte es einer diesbezüglichen Ausnahme von $\$ 250$ StPO in den anschließenden Vorschriften, die de lege lata aber ge- rade nicht besteht, wenn man einmal von der Möglichkeit der Verlesung im allseitigen Einverständnis absieht $(\mathbb{S} 251$ Abs. 1 Nr. 1 StPO). Dazu kann es durchaus kommen, weil sie dem Opfer die möglicherweise psychisch belastende Aussagesituation erspart und der Angeklagte daran ein Interesse haben kann, weil dies regelmäßig strafmildernd wirkt. ${ }^{61}$ Im Übrigen aber erfolgt der Schutz des Stalking-Opfers im Prozess vor dem Angeklagten nach geltendem Recht allenfalls über $\mathbb{\int} \int 247,247$ a StPO. Dabei könnten Ausnahmen vom strafprozessualen Unmittelbarkeitsgrundsatz, wie er sich de lege lata in $\mathbb{2 5 0}$ StPO niederschlägt und Einschränkungen in den folgenden Vorschriften erfährt, aus Gründen des Opferschutzes durchaus gerechtfertigt werden. ${ }^{62}$ Des Weiteren muss bedacht werden, dass es einigermaßen widersprüchlich auf das Opfer wirken muss oder jedenfalls kann, dass das - als „lästige und manchmal als entwürdigend empfundene "63 - Stalking-Tagebuch einerseits erforderlich, andererseits aber zugleich unverwertbar ist. Im Moment stehen solche Überlegungen aber nicht auf der Agenda des (Reform-)Gesetzgebers. Stattdessen sollen andere Wege beschritten werden.

\section{Bestellung eines Opferanwalts}

Der Bundesrat hat den „Entwurf eines Gesetzes zur Verbesserung des Schutzes der Opfer von Zwangsheirat und schwerem Stalking " auf den Weg gebracht. ${ }^{64}$ Er sieht eine Ergänzung des $\ 397$ a StPO und damit die Bestellung eines Rechtsanwalts, des sog. Opferanwalts, für den Nebenkläger vor. Nach dem Entwurf soll der Gestalkte, sofern er als Nebenkläger auftritt, auf Antrag einen solchen Rechtsbeistand bestellt bekommen, wenn ein Fall des $\$ 238$ Abs. 3 StGB vorliegt. Wenn der Gestalkte unter 16 Jahre alt ist oder auf Grund seiner psychischen oder physischen Situation „seine Interessen ersichtlich nicht selbst ausreichend wahrnehmen kann “ 65 , soll dies bereits gelten, wenn „bloß“ $\$ 238$ Abs. 2 StGB in Rede steht. Bei $\$ 397$ a Abs. 2 StPO (Gewährung von Prozesskostenbeihilfe) verbleibt es hingegen, wenn lediglich der Stalking-Grundtatbestand des $\mathbb{} 238$ Abs. 1 StGB angeklagt ist. „Diese Differenzierung nach dem Grad der Betroffenheit gewährleistet einen den Belangen des Opfers im Einzelfall angemessen und flexibel Rechnung tragenden Schutz “66, führt der Bundesrat als Argument für die unterschiedliche Behandlung von Stalking-Opfern an.

Interessant ist die Begründung, warum man sich zu diesem Schritt entschieden hat. Sie wird in den Motiven nicht weniger als dreimal bemüht und lautet an der entscheidenden Stelle wie folgt ${ }^{67}:$, $\$ 397 \mathrm{a}$ StPO schützt Verletzte, die Opfer von erheblichen Angriffen auf fundamentale Persönlichkeitsrechte, namentlich ihren höchstpersönlichen Lebensbereich oder ihr Leben geworden sind, so dass sie regelmäßig besonders traumatisiert und daher in besonderem Maße schutzbedürftig sind. Darüber hinaus kommt der Zengenaussage solcher Opfer in Anbetracht der gerade bei Sexual-und Beziehungstaten häufig anzutreffenden "Aussage-gegen-Aussage "Situation regelmäßig besondere Bedeutung zu. Vor diesem Hintergrund sehen sie sich auch oftmals einer besonders kritischen Prüfung ihrer Glaubwürdigkeit ausgesetzt. "Insofern geht der Bundesrat, weil er diese Voraussetzungen bei Opfern von schwerem Stalking als gegeben ansieht, implizit davon aus, dass an der Vernehmung des Stalking-Opfers in der Hauptverhandlung kein Weg vorbeiführt.

Als Schritt in Richtung Opferschutz ist der Entwurf dennoch zu begrüßen. Man darf sich berechtigte Hoffnungen machen, dass er realisiert wird. Die Bundesregierung hat in der Stellungnahme zum Entwurf jedenfalls bekundet ${ }^{68}$, dass es ein „stetes Anliegen“ sei, die Situation der Opfer von Straftaten zu verbessern. Von daher wür- 
den alle Maßnahmen unterstützt, „die unter Beachtung berechtigter Belange der anderen Verfahrensbeteiligten das Ziel verfolgen, die Interessen der Opfer noch stärker zu berücksichtigen“. Angesichts dessen begrüßt man es, „dass die Länder - trotz der damit für ihre Haushalte verbundenen Mehrausgaben - Regelungsvorschläge zur Verbesserung des Opferschutzes unterbreiten“, wobei der Offerte zugestimmt wird, „für Opfer von Zwangsheirat und bestimmten schweren Formen des Stalkings den Zugang zu einem kostenlosen Opferanwalt zu erweitern“. Wenngleich die Bundesregierung abschließend „hinsichtlich der Ausgestaltung beider Vorschriften im Einzelfall noch Prüfbedarf“ ausmacht, steht sie dem Vorhaben insofern äußerst wohlwollend gegenüber. Dass sich die Regierungsfraktionen im Bundestag anders verhalten, steht eher nicht zu erwarten, sodass der Entwurf des Bundesrats mit einer relativ hohen Wahrscheinlichkeit einmal im Bundesgesetzblatt stehen wird. Ob sich damit die Situation von Stalking-Opfern merklich verbessert, steht freilich auf einem anderen Blatt Papier.

\section{Fazit}

Ob weitere rechtspolitische Vorhaben, um zu einem Fazit zu kommen, angebracht sind, wie etwa die Möglichkeit der Verlesung eines Stalking-Tagebuchs, bleibt abzuwarten. Einstweilen müssen die Fälle aber überhaupt erst einmal zur Anklage gebracht werden, um abschätzen und (empirische) Erfahrungen machen zu können, wie sich die Täter-Opfer-Situation in der Hauptverhandlung darstellt. Wenn einmal diesbezüglicher Reformbedarf besteht, wird der Gesetzgeber die geschilderte Möglichkeit in Betracht zu ziehen und deren Sinn und Zweck (Opferschutz) gegen die Interessen des Angeklagten sowie die Belange des strafprozessualen Unmittelbarkeitsgrundsatzes abzuwägen haben. Im Moment aber müsste es überhaupt erst einmal zur strafgerichtlichen Aufarbeitung von Stalking-Fällen kommen. \238 Abs. 4 StGB und $\$ 376$ StPO stehen dabei nicht im Wege. Sie bilden keinesfalls ein unüberwindbares prozessuales Hemmnis bei der Strafverfolgung von (sog. rejected) Stalkern.

\section{Matthias Krüger ist wissenschaftlicher Assistent in Halle}

\section{Fußnoten:}

1 Fünfsinn, NK 2005, $82 \mathrm{ff}$

2 Gerhold, NK 2007, 2, 3.

3 Freudenberg, NK 2005, 84 ff. Ebenso Rackow, GA 2008, 552, 568.

4 Frommel, NK 2005, 86 ff.; dies., NK 2006, 109.

5 Dies betont bereits Frommel, NK 2005, 86, 87.

6 S. zu Folgendem BT-Drs. 16/6308, S. 382 f.

7 Vgl. zu dieser Sorge etwa Frommel, NK 2006, 109.

8 Ebda.

9 Quelle: NJ 2008, 300, 301.

10 Quelle: NJ 2008, 253, 254.

11 Gazeas, KJ 2006, 247, 268

12 Neubacher, ZStW 118 (2006), 855, 867.

13 Eiden, ZIS 2008, 123, 128. Vgl. ebenso bereits Neubacher/Seher, JZ 2007, 1029, 1030: „Der kriminalpolitische Spagat zwischen Verfassungsrecht und Sicherheitsversprechen läuft jedenfalls Gefahr, weder die verfassungsrechtlichen Grenzen von Strafgesetzgebung zu beachten noch die Erwartungen der betroffenen Opfer an effektiven Schutz zu erfüllen. Es droht dann, gleichsam als Preis für eine symbolische Gesetzgebung, eine Erosion des Vertrauens in das Recht. Dieser Gefahr ist auch der neue Straftatbestand der beharrlichen Nachstellung ausgesetzt."

14 Knauer/Reinbacher, StV 2008, 377, 379. Vgl. hierzu, wenngleich aus anderen Gründen bereits Krüger, NJ 2008, $150 \mathrm{ff}$.

15 Kinzig/Zander, JA 2007, 481, 487 und ferner noch Rackow, GA 2008, 552, 567: „\$2 238 StGB bildet kein gelungenes Modell.“

16 Eiden, ZIS 2008, 123.

17 S. dazu und zu weiteren Zahlen Krüger in: Sackmann/Kison/Horn (Hrsg.), Graffiti kontrovers - Ergebnisse der ersten mitteldeutschen Graffitistudie, 2008, S. 50, 53.

18 LG Kiel NStZ 2008, 219.
$19 \mathrm{Ob}$ in solchen Konstellationen ein Betrug vorliegt, ist umstritten, vgl. die Nachw. bei Müller in: Krüger, Stalking als Straftatbestand, 2007, S. 56 sowie ferner Gerhold, NK 2007, 2, 3. Im zugrunde liegenden Fall konnte man es wohl deshalb (eher als sonst) annehmen, weil sich der Angekl. keine Gedanken darüber machte, „wer die anfallenden Kosten bzw. vertragsgemäßen Gegenleistungen (Kaufpreis oder Wert) tragen würde“ und er selbst „weder zahlungswillig noch zahlungsfähig“ war, vgl. LG Kiel NStZ 2008, 219, 220.

$20 \mathrm{Ob}$ solche Fälle de lege ferenda überhaupt unter Stalking-Strafvorschriften fallen (sollen), wird noch nicht in Deutschland, wohl aber im (österreichischen) Ausland diskutiert, s. hierzu die Nachw. bei Seling, $\mathbb{1} 107$ a StGB - Eine Strafvorschrift gegen Stalking, 2006, S. 77. Zum inhaltlichen Gesichtspunkt dafür, dass es sich um Stalking handelt, könnte man machen, dass es ohne wirklichen sachlichen Anlass um die Person des Nachbarn als solchen geht, auf die man - wie generell beim Stalking - fixiert ist, wohingegen Nachbarschaftszwistigkeiten, deren Ursachen in $\mathbb{S} \mathbb{S} 907 \mathrm{ff}$. BGB liegen, kein Stalking im soziologischen Sinne darstellen, insbesondere wenn sie von beiden Seiten ausgehen. De lege lata kommt es für $\mathbb{} 238$ StGB dagegen bloß darauf, ob sein Wortlaut erfüllt ist, weil die Motive für kriminelles Verhalten allenfalls für die Strafzumessung von Interesse sind.

21 Vgl. dazu etwa BGHSt 32, 38, 41 ff.

22 Die bloßvorübergehende Einnahme von Medikamenten reicht für eine (gefährliche) Körperverletzung in mittelbarer Täterschaft, wohingegen von einer „schwerwiegenden Beeinträchtigung der Lebensgestaltung“ in solchen Fällen noch nicht zwangsläufig gesprochen werden kann, wenngleich es umstritten ist (vgl. einerseits Mitsch, JURA 2007, 401, 406 und andererseits Krüger, NJ 2008, 150, 153 in Fn. 36). Bei einer dauerhaften Einnahme von Medikamenten kann sich dies freilich anders darstellen, insbesondere wenn damit erhebliche Nebenwirkungen und Langzeitfolgen oder sogar die Gefahr der Abhängigkeit einhergehen. In dieser Hinsicht kommt es wiederum auf den jeweiligen Einzelfall an, insbesondere weisen nicht sämtliche Psychopharmaka ein entsprechendes Suchtpotenzial auf. Dabei werden selektive Serotonin-Wiederaufnahme-Hemmer (SSRIs) bei posttraumatischen Belastungsstörungen (infolge von Stalking) eher als klassische trizyklische Antidepressiva verschrieben, weil SSRIs gegenüber diesen weniger toxisch wirken, wohingegen vom Einsatz angstlösender Benzodiapine zur Angstbehandlung bei Stalking-Opfern eher abgeraten wird, weil deren längerer Gebrauch eine Missbrauchs- oder Abhängigkeitsentwicklung begünstigt [vgl. zum Ganzen aus medizinischer Perspektive etwa Kühner/Weiß in: Dreßing/ Gass (Hrsg.), Stalking, S. 96]. Gleiches gilt für Schlafmittel, die im weitesten Sinne ebenfalls zu Psychopharmaka zählen und möglicherweise vom Nebenkl. im Falle des LG Kiel wegen seiner „massiven Einschlafstörungen“ eingenommen worden sind. Sie bergen gleichfalls die nicht unerhebliche Gefahr in sich, bei längerem Gebrauch zu einer Abhängigkeit zu führen.

23 Kinzig/Zander, JA 2007, 481, 485. Vgl. im selben Sinne Steinberg, JZ 2006, 30, 31 (bedenkliche Anzahl unbestimmter Rechtsbegriffe); Eiden, ZIS 2008, 123, 126 (Bündel unbestimmter Rechtsbegriffe); Rackow, GA 2008, 552, 561 (Vielzahl komplex verschränkter wertausfüllungsbedürftiger Begriffe) und ferner bereits Frommel, NK 2005, 86; Gerhold, NK 2007, 2.

24 Freudenberg, NJ 2006, 535, 537; dies., NK 2005, 84, 85.

25 S. hierzu Krüger, Stalking als Straftatbestand, S. 109 ff. (zu „räumliche Nähe“), S. 139 ff., 152 ff. (zu $\$ 238$ Abs. 1 Nr. 5 StGB), S. 160 ff. (zu „beharrlich“) sowie S. $171 \mathrm{ff}$. (zu „schwerwiegend“).

26 Nach Thoss, StV 2006, 160, 162 enthält $\mathbb{3 0 3}$ Abs. 2 StGB einen „unbestimmten objektiven Straftatbestand“ mit einer „generalklauselartigen Tathandlung“, wobei insbesondere das Merkmal „nicht nur unerheblich“ die „Reichweite des Tatbestands [...] noch unbestimmter“ macht. Ohne weitere Begründung vermag die Kritik indes nicht zu überzeugen, vgl. dazu näher Krüger, Graffiti - Juristische Aspekte des $\$ 303$ Abs. 2 StGB und deren Bedeutung für polizeiliche Ermittlungen wegen Graffiti, Ascherslebener Manuskripte, 2006, S. 15.

27 In diesem Sinne etwa Frommel, NK 2005, 86, 87; Gerhold, NK 2007, 2 3

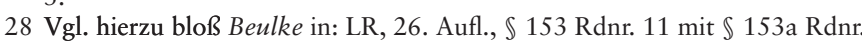
17

29 Gerhold, NK 2007, 2, 3.

$30 \mathrm{Vgl}$. dazu bloß I. Wolf, Graffiti als kriminologisches und strafrechtsdogmatisches Problem, 2004, S. 102.

31 Hirsch in: LK, 11. Aufl., $\mathbb{\$} 230$ Rdnr. 16 f.; Stree in: Schönke/Schröder, 27. Aufl., $\mathbb{} 230$ Rdnr. 3; Paeffgen in: NK, 2. Aufl., $\$ 230$ Rdnr. 14 ff.; Horn/ Wolters in: SK, $\mathbb{} 230$ Rdnr. 4 - jeweils m. w. N.

32 BVerfGE 51, 176, 187; RGSt 77, 17, 20; 77, 72, 73; BGHSt 16, 225, 228 ff.; BayObLG NJW 1991, 1765; BVerwG NJW 1959, 448, 449; Fischer, 55. Aufl., $\$ 230$ Rdnr. 3; Lackner/Kühl, 26. Aufl., \$ 230 Rdnr. 5 sowie - wenngleich bereits etwas differenzierend - Hardtung in: MünchKommStGB, $\$ 230$ Rdnr. 45 ff. Vgl. für $\$ 376$ StPO in diesem Sinne etwa OLG Oldenburg GA 1959, 187; Meyer-Goßner, 51. Aufl., $\mathbb{S} 376$ Rdnr. 7; Stöckel in: KMR, $\mathbb{} 376$ Rdnr. 9 ff.; Hilger in: LR, 25. Aufl., $\$ 376$ Rdnr. 15.

33 BT-Drs. 16/3664, S. 1 unter 1.

34 Ebda., S. 3. 
35 Abg. Schewe-Gerigk, BT-Plenarprotokoll 16/70 vom 30. November 2006, S. 6955.

36 Abg. Wunderlich, BT-Plenarprotokoll 16/70, S. 6953.

37 Abg. Lambrecht (SPD), BT-Plenarprotokoll 16/70, S. 6956.

38 Freudenberg, NJ 2006, 535, 538.

39 BT-Drs. $16 / 575$, S. 8.

40 Mitsch, NJW 2007, 1237, 1241.

41 Mitsch, NJW 2007, 1237, 1241 sowie ferner noch Mosbacher, NStZ 2007, 665,670 , der insofern zwar $\$ 238$ Abs. 4 StGB für sachgerecht hält, zugleich aber die Einstufung als Privatklagedelikt wegen des damit für das Opfer verbundenen Kostenrisikos für nicht ganz glücklich hält und anzweifelt.

42 Vgl. zu den folgenden Zitaten jeweils Buettner, ZRP 2008, 124 ff. Zust. Rackow, GA 2008, 552, 568 in Fn. 125.

43 BT-Drs. 16/3663, S. 2.

44 Buettner, ZRP 2008, 124 (Hervorhebung nicht im Original).

45 Vgl. die Zahlen bei $V o ß$, Stellungnahme zur öffentlichen Anhörung im Rechtsausschuss vom 18. Oktober 2006 zum Thema „Stalking“ unter 3. (Beziehungskonstellationen Opfer-Täter).

46 S. zur Einteilung von Stalker-Typen und deren Motiven näher Müller in: Krüger, Stalking als Straftatbestand, S. 25 ff. m. w. N. insbesondere zum soziologischen Schrifttum. Dass diese Einteilung im Rahmen der vorliegenden (Teil-)Problematik durchaus von Interesse ist, wurde an gleicher Stelle dargetan, vgl. Krüger, Stalking als Straftatbestand, S. 198.

47 Buettner, ZRP 2008, 124, 125 (Hervorhebung nicht im Original).

48 Obwohl Nr. 86 RiStBV bloß das „öffentliche Interesse“ im Sinne von \$ 376 StPO näher erläutert, können deren Begriffserläuterungen - trotz der unterschiedlichen Begrifflichkeit - durchaus auf das „besondere öffentliche Interesse“ gemäß $\$ 238$ Abs. 4 StGB übertragen werden. Im Bereich der einfachen und fahrlässigen Körperverletzungsdelikte geht man jedenfalls von einer weitgehenden Identität beider Begriffe aus, wie die Lektüre der Nr. 233 ff. RiStBV zeigt.

49 Mitsch, NJW 2007, 1237, 1241 sowie ferner noch Mosbacher, NStZ 2007, 665, 670 und im selben Sinne bereits Fünfsinn, NK 2005, 82, 83.

50 In dieser Richtung, wenngleich es sich dabei um ein generelles Problem bei Antragsdelikten handelt, bereits Janovsky, Stellungnahme zur öffentlichen Anhörung im Rechtsausschuss vom 18. Oktober 2006 zum Thema „Stalking“, S. 4: „Beeinflussungen durch den Täter“ sowie Hecht, Berliner Interventionszentrale bei häuslicher Gewalt, Stellungnahme zur öffentlichen Anhörung im Rechtsausschuss vom 18. Oktober 2006 zum Thema „Stalking“, S. 12: „Einflussnahme des Täters auf das Opfer“.

51 Esser/Wolmerath, Mobbing, 2. Aufl., S. 292; Bieszk/Sadtler, NJW 2007, 3382, 3383.

52 Vgl. dazu Mühe, Mobbing am Arbeitsplatz - Strafbarkeitsrisiko oder Strafbarkeitslücke, 2006, S. 55 f.; Bieszk/Sadtler, NJW 2007, 3382, 3386 f. sowie - auf Basis des $\$ 238$ Abs. 1 Nr. 5 StGB - ferner noch Krüger, Stalking als Straftatbestand, S. $148 \mathrm{f}$.

53 Hoffmann, Stalking, S. 162.

54 Gass in: Dreßing/Gass (Hrsg.), Stalking, S. 73.
55 S. hierzu bloß Krüger, Stalking als Straftatbestand, S. 164 ff. m. w. N.

56 BVerfGE 80, 367, 379; BGHSt 19, 325, 332 ff.; 34, 397, 401; Beulke, Strafprozessrecht, 10. Aufl., $\mathbb{2} 23$ Rdnr. 473; Hellmann, Strafprozessrecht,

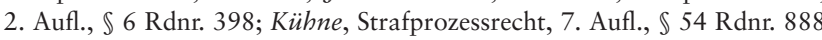
- jeweils m. w. N.

57 BVerfGE 80, 367, 381

58 Nach Geppert, Grundsatz der Unmittelbarkeit im Strafverfahren, 1979, S. 181 (Hervorhebung im Original) soll zwar jener zentrale $\$ 250 \mathrm{StPO}$ nicht schlechthin Sitz des Unmittelbarkeitsprinzips sein, sondern bloß einen Teilbereich von (materieller) Unmittelbarkeit regeln. Gleichwohl lässt sich aber nicht ernsthaft bestreiten, dass die Norm eine gesetzliche Ausprägung dieser Verfahrensmaxime ist.

59 BGHSt 6, 141, 143; 20, 160, 161; BGH NStZ 1982, 79; Meyer-Goßner, \250 Rdnr. 8; Gollwitzer in: LR, 25. Aufl., \ 251 Rdnr. 7; Schneidewin, JR 1951, 481, 483; Wömpner, NStZ 1983, 293, 294. Wenn man mit der Gegenansicht eine Unterscheidung zwischen zu Beweiszwecken angefertigten und anderweitig hergestellten „schriftlichen Erklärungen“ von vornherein nicht zulassen will (s. dazu etwa Krause, Urkundenbeweis im Strafprozeß, 1966, S. 154 ff., 159), kommt $\$ 250$ Satz 2 StPO erst Recht zum Zuge.

60 Meyer-Goßner, $\mathbb{} 250$ Rdnr. 8; Schneidewin, JR 1951, 481, 483.

61 BGH GA 1962, 339; Stree in: Schönke/Schröder, $\$ 46$ Rdnr. 41; Franke in: MünchKommStGB, $\mathbb{S} 46$ Rdnr. 48, wohingegen der umgekehrte Schluss nicht zulässig ist, vgl. dazu bloß BGH, a. a. O. sowie StV 1987, 100.

62 Bestimmte Ausnahmen vom Unmittelbarkeitsprinzip werden jedenfalls mit Opferschutz begründet, wie es insbesondere für die Videovernehmung und deren gesetzliche Lozierung in $\$ 255$ a StPO zutrifft, vgl. dazu etwa Mildenberger, Schutz kindlicher Zeugen im Strafverfahren durch audiovisuelle Medien, 1995, S. 230 ff.; Nowak, Der gefährdete Zeuge im deutschen und polnischen Strafverfahren, 2000, S. 72 ff.; Swoboda, Videotechnik im Strafverfahren, 2002, S. 399 ff. Gerade die Videovernehmung des StalkingOpfers könnte sich unter Opferschutzgesichtspunkten anbieten. Wenn man sich dabei noch vor Augen führt, dass der Bundesrat in seinem - sogleich noch darzustellenden - Gesetzentwurf zur Einführung eines Rechtsbeistands für Opfer von schwerem Stalking diesen in einem Atemzug mit den

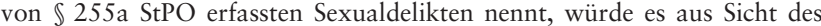
Gesetzgebers durchaus Sinn machen, wenn er zu einer entsprechenden Novellierung dieser Vorschrift schreiten würde.

63 Gass in: Dreßing/Gass (Hrsg.), Stalking, S. 73. Vgl. ferner Hoffmann, Stalking, S. 162: Dokumentation des Geschehens als eine oft nicht unbeträchtliche Belastung für die Opfer.

64 BT-Drs. 16/9448.

65 Meyer-Goßner, $\mathbb{S} 397$ a Rdnr. 3 a. E., wie es bei Stalking-Opfern aus denselben Gründen, warum das (besondere) öffentliche Interesse anzunehmen ist, durchaus nahe liegt.

66 BT-Drs. 16/9448, S. 7.

67 Ebda., S. 6 (Hervorhebung nicht im Original).

68 Vgl. zum Folgenden jeweils BT-Drs. 16/9448, S. 8.

\section{Nachträgliche Sicherungsverwahrung - eine empirische erste Bilanz}

Michael Alex

\section{Ausgangslage}

Am 29.07.2004 wurde mit dem $\$ 66b StGB die nachträgliche Sicherungsverwahrung im deutschen Strafrecht eingeführt, nachdem bereits seit 2001 einzelne Bundesländer (Baden-Württemberg, Bayern, Sachsen-Anhalt, Niedersachsen, Thüringen) polizeirechtliche Regelungen zur Unterbringung im Strafvollzug nach vollständiger Strafvollstreckung getroffen hatten. Diese Ländergesetze hatte das Bundesverfassungsgericht am 10.02.2004 wegen Verstoßes gegen Art. 74 GG für verfassungswidrig erklärt und dem Bundesgesetzgeber empfohlen, bis 30.09.2004 eine strafrechtliche Lösung zu finden. Die Regelung in $\ 66 \mathrm{~b}$ StGB geht infolge ihrer Dauerwirkung deutlich weiter als die von den Nationalsozialisten in Art. 5 des „Gewohnheitsverbrechergesetzes“ vom 24.11.1933 (RGBl 1933, 995) getroffene Übergangslösung für Verurteilungen aus der Weimarer Republik.

Vier Jahre nach Inkrafttreten des $\$ 66 \mathrm{~b}$ StGB kann eine erste Bilanz gezogen werden. Nach den vorliegenden Unterlagen und veröffentlichten Entscheidungen sind von 2001 (zunächst auf Grundlage von Straftäter-Unterbringungsgesetzen einzelner Bundesländer) bis Ende 2006 gut 110 Anträge auf Anordnung der nachträglichen Unterbringung durch die Gerichte zurückgewiesen worden, darunter knapp 40, die auf Grundlage der Straftäter-Unterbringungsgesetze 